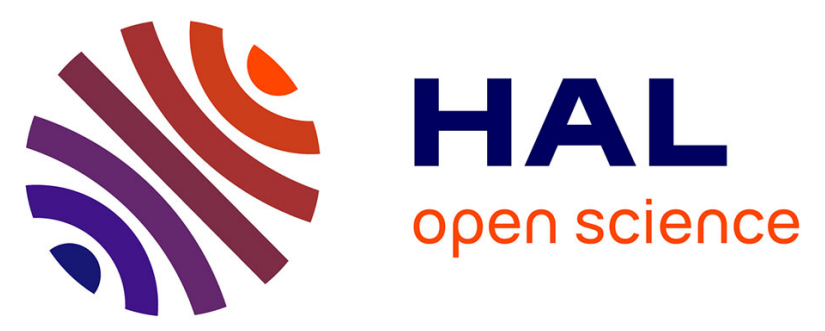

\title{
Rythme d'apparition des racines primaires du maïs (Zea mays L.). II. - Variations observées sur quelques génotypes
}

\author{
Claude Varlet-Grancher, Maurice Derieux, Marie-Odile Jordan, Philippe \\ Girardin, Didier Picard
}

\section{To cite this version:}

Claude Varlet-Grancher, Maurice Derieux, Marie-Odile Jordan, Philippe Girardin, Didier Picard. Rythme d'apparition des racines primaires du maïs (Zea mays L.). II. - Variations observées sur quelques génotypes. Agronomie, 1987, 7 (9), pp.695-702. hal-00885043

\section{HAL Id: hal-00885043 \\ https://hal.science/hal-00885043}

Submitted on 1 Jan 1987

HAL is a multi-disciplinary open access archive for the deposit and dissemination of scientific research documents, whether they are published or not. The documents may come from teaching and research institutions in France or abroad, or from public or private research centers.
L'archive ouverte pluridisciplinaire HAL, est destinée au dépôt et à la diffusion de documents scientifiques de niveau recherche, publiés ou non, émanant des établissements d'enseignement et de recherche français ou étrangers, des laboratoires publics ou privés. 


\title{
Rythme d'apparition des racines primaires du maïs (Zea mays L.). II. - Variations observées sur quelques génotypes
}

\author{
Claude VARLET-GRANCHER, Maurice DERIEUX*, Marie-Odile JORDAN**, Philippe GIRARDIN** \\ \& Didier PICARD*** \\ I.N.R.A., Station d'Ecophysiologie des Plantes fourragères, Centre de Recherches de Poitou-Charentes, \\ F-86600 Lusignan \\ * I.N.R.A., Station d'Amélioration des Plantes, Centre de Recherches de Lille, Estrées-Mons-en-Chaussée, \\ F-80200 Péronne \\ ** I.N.R.A., Station d'Agronomie, Centre de Recherches de Colmar, B.P. 507, F-68021 Colmar \\ *** I.N.R.A., Département d'Agronomie, Centre de Recherches de Paris-Grignon, F-78850 Thiverval-Grignon
}

\begin{abstract}
Une étude de la structure du système racinaire a été présentée dans 2 articles précédents ; ici nous avons observé les variations de quelques paramètres caractéristiques pour différents génotypes à partir d'un essai diallèle (6 lignées sans hybrides réciproques) plus un essai variétal (4 variétés) dans le nord de la France et d'un essai variétal ( 3 variétés) au Canada.

Dans les essais variétaux, le nombre de racines allongées des premiers entre-nœuds paraît plus fortement lié au génotype mais une liaison avec la précocité n'a pu être établie que pour les observations effectuées dans le nord de la France. Pour les entre-nœuds supérieurs, l'influence du milieu semble plus importante et il peut y avoir des effets de compensation entre tous les entre-nœuds conduisant ainsi à des nombres totaux de racines peu différents. Toutefois ces effets n'ont pas été observés dans l'essai diallèle.

La relation entre nombre de racines et résistance à la verse racinaire n'est pas très forte mais encourageante pour le sélectionneur ; l'importance de l'Aptitude Générale à la Combinaison (A.G.C.) pour le nombre de racines sur les premiers entre-nœuds permet une sélection indépendante du testeur à un stade précoce.

La vitesse d'apparition des racines et la relation entre l'émission racinaire et l'émission foliaire varient peu avec le génotype mais pourraient dépendre du milieu.
\end{abstract}

Mots clés additionnels : Phyllochrone, somme de températures, entre-noeuds.

The structure of the maize root system was studied in two previous papers and we have studied here the variation of some typical parameters with genotype. Results are given of a diallel trial $(6$ inbreds without reciprocal hybrids) and two varietal trials. Root number on the first internodes has been related to genotype, and a good relationship with earliness was established. For the next internodes environmental effects were more important and the compensation between the different internodes resulted in low variation of total root number. However this result was not observed in the diallel trial. Relationships between root number and resistance to lodging was not very high but encouraging for the breeder. The high General Combining Ability of the first internode root number would allow breeding with any testor at an early stage. The rate of appearance and relationship between root appearance and leaf appearance were not modified by genotype but seemed to depend on environmental conditions.

Additional key words : Internodes, phyllochrone, sum of temperatures.

\section{INTRODUCTION}

Le rythme d'émission des racines primaires du maïs a été décrit avec précision pour la variété « DEA » à Colmar (PICARD et al., 1985). Une autre étude (JoRDAN $e t$ al., 1988) a porté sur la variabilité observable avec différentes conditions de culture.
A partir des observations effectuées sur quelques variétés, nous avons essayé ici, d'établir dans quelle mesure certains paramètres caractéristiques de l'enracinement pouvaient dépendre du génotype. En effet, la relation entre les cinétiques d'apparition des racines et des feuilles par exemple, dépend fortement du génotype chez le riz (PICARD, 1984) alors qu'elle sem- 
ble peu variable pour le blé (PINTHUS, 1969 ; KLEPPER et al., 1984).

Le choix des variétés utilisées dans cette étude a été fait sur la précocité de manière à représenter au mieux (avec le minimum de traitement) la gamme de génotypes cultivés en France et dans une moindre mesure au Canada.

La culture d'hybrides de maïs de plus en plus précoces se heurte à de nombreuses difficultés (DERIEUX, 1978) et notamment à celles résultant de la sensibilité de ces génotypes à la verse en « végétation ». Celle-ci, appelée aussi «verse racinaire », résulte de la mauvaise capacité du système racinaire à maintenir la plante dressée ; elle intervient en cours de végétation ou de maturation et doit être distinguée de la verse à maturité qui résulte de la pourriture des tiges (BARRIERE, 1979). Nous avons donc aussi mis en observation des hybrides expérimentaux issus de lignées précoces qui correspondent à une large gamme de variations pour le caractère verse en végétation. A partir de cet essai, nous avons essayé de relier cette verse aux observations faites sur le système racinaire et d'obtenir quelques informations sur le mode de transmission de ce caractère.

\section{MATÉRIEL ET MÉTHODES}

Trois essais ont été mis en place ; l'un à Guelph à la station expérimentale Elora (Ontario, Canada) et les 2 autres à la station d'Amélioration des Plantes d'Estrées-Mons (dans le nord de la France). Ils comportaient 4 répétitions et ont été conduits selon les conditions habituelles de la grande culture décrites précédemment (PICARD et al., 1985).

A Guelph $\left(43^{\circ} 7 \mathrm{~N}, 82^{\circ} 1 \mathrm{O}\right.$, altitude $\left.200 \mathrm{~m}\right)$ l'essai variétal a été semé le 17 mai 1984 à la densité de $75000 \mathrm{pl} /$ ha et comportait 3 hybrides : LG 1, hybride très précoce, SX 111 hybride précoce et $\mathrm{P} 3906$ hybride demi-tardif.

A Mons (désignation du site Estrées-Mons; $49^{\circ} 49 \mathrm{~N}, 2^{\circ} 01 \mathrm{E}$, altitude $98 \mathrm{~m}$ ) le premier essai compte 4 hybrides semés le 6 mai 1983 à la densité de $80000 \mathrm{pl} / \mathrm{ha}: \mathrm{F} 7 \times \mathrm{F} 2$ hybride très précoce à grains cornés; DEA hybride corné denté, demi-précoce ; MONARQUE INRA 282, hybride corné demi-précoce ayant tendance à la prolificité en épis et INRA 508, hybride denté demi-tardif. Le second essai est un diallèle sans hybrides réciproques ni lignées. Les conditions de culture sont les mêmes que pour l'essai précédent et les caractéristiques des 6 lignées utilisées sont les suivantes:

* F2 : lignée cornée précoce, donnant des hybrides résistants à la verse, utilisée dans la quasi totalité des hybrides cultivés au nord de la Loire,

* F257 : lignée cornée précoce, donnant des hybrides dont la résistance à la verse dépend essentiellement des combinaisons. Elle transmet une excellente vigueur au départ,

* F244 : lignée dentée précoce, donnant des hybrides très résistants à la verse et possédant une excellente vigueur au départ,

* F250 : lignée dentée précoce, donnant de bons hybrides résistants à la verse racinaire mais sensibles à la pourriture des tiges,

* F252 et F272 : lignées dentées précoces, donnant des hybrides d'excellente valeur agronomique mais dont la résistance à la verse n'est pas toujours suffisante.

Ces lignées ont été choisies d'après les observations préliminaires dont nous disposions pour :

- leur aptitude à donner de bons hybrides,

- leur sensibilité très différente à la verse,

- et leur nombre de racines également très différent.

La recherche de liaison entre ces deux derniers caractères était l'objectif principal de l'essai. Plusieurs lignées sont apparentées : F2 est un des grand-parents de F257-F244, F252 et F272 ont un parent commun F186.

Les caractéristiques générales des sols et des climats ont été données par ailleurs (JORDAN et al., 1988) et ne sont que brièvement rappelées ici. A Mons les essais sont implantés sur un sol de limon très profond $(10 \mathrm{~m}$ environ) de texture limono-argileuse et présentant unc réserve utile importante (150 à $200 \mathrm{~mm}$ par $\mathrm{m}$ de sol). Le sol de Guelph est également un sol de limon profond $(2$ à $3 \mathrm{~m})$ à texture limono-sableuse et à bonne réserve utile ( 140 à $170 \mathrm{~mm}$ par $\mathrm{m}$ de sol). Les climats sont caractérisés par les données classiques relevées par des postes météorologiques situés près des essais.

Les observations biologiques sont faites sur 20 plantes ( 5 par répétition) selon la méthode décrite dans le premier article (PICARD et al., 1985). A Mons les prélèvements ont été effectués tous les 10 jours jusqu'à floraison pour l'essai variétal et trois fois avant floraison pour l'essai diallèle puis à la récolte pour les deux essais. A Guelph les déterminations ont été faites toutes les semaines jusqu'à la floraison.

Pour chaque plante nous avons compté (voir PICARD et al., 1985) :

- le nombre de feuilles visibles et étalées (ligule dégagée),

- le nombre d'entre-nœuds portant des racines adventives,

- et pour chaque entre-nœud le nombre de racines primaires allongées (longueur supérieure à $3 \mathrm{~cm}$ ).

La verse en végétation ou racinaire est déterminée comme le pourcentage des plantes inclinées à plus de $45^{\circ}$ et dénombrées en cours de maturation (30 à 40 jours après la floraison). La verse par pourriture est obtenue selon la méthode décrite par BARRIÈRE (1979).

La production de grain a été mesurée à la récolte pour les deux essais de Mons sur des parcelles réservées à cet effet.

La comparaison statistique des moyennes a été faite selon la méthode de NEWMAN et KEuls (DAGNELIE, 1970) sur certains des résultats présentés.

Soulignons que ces dispositifs ne permettent pas d'étudier les interactions génotype $\times$ milieu mais visent seulement à comparer le comportement de quelques génotypes dans un même milieu considéré dans son ensemble et déterminé par le site, l'année et les conditions de culture.

\section{RÉSULTATS ET DISCUSSION}

\section{A. Comportement général des essais}

Dans les essais variétaux, les dates de floraison s'étalent sur 15 jours à Guelph comme à Mons (tabl. 1a) 
TABLEAU 1a

Comportement général des essais variétaux à Mons et à Guelph. Les sommes de température de l'air sont calculées avec un seuil $6{ }^{\circ} \mathrm{C}$. Observations and agronomic characteristics of varieties studied at Mons and Guelph. Temperature sum with base temperature $6{ }^{\circ} \mathrm{C}$.

\begin{tabular}{|c|c|c|c|c|c|c|c|c|c|}
\hline & \multicolumn{4}{|c|}{ Floraison } & \multicolumn{5}{|c|}{ Recolte (13 octobre 1983) } \\
\hline & Date & $\Sigma T>6$ & $\begin{array}{l}\mathrm{Nb} \text { de } \\
\text { feuilles }\end{array}$ & $\begin{array}{l}\text { Nb racines } \\
>3 \mathrm{~cm}\end{array}$ & $\Sigma T>6$ & $\begin{array}{l}\mathrm{Nb} \text { racines } \\
>3 \mathrm{~cm}\end{array}$ & $\begin{array}{c}\text { Rendement } \\
\text { t/ha }\end{array}$ & $\begin{array}{l}\text { Verse raci- } \\
\text { naire } \%\end{array}$ & $\begin{array}{l}\text { Verse pour- } \\
\text { riture } \% 0\end{array}$ \\
\hline \multicolumn{10}{|l|}{ Mons } \\
\hline$F 7 \times F 2$ & 25.07 & 786 & $13,2 \mathrm{a}$ & 25,2 a $(0,18)$ & 1643 & 30,9 a $(0,18)$ & 5,2 & 20 & 45 \\
\hline DEA & 29.07 & 849 & $15,3 \mathrm{~b}$ & 32,2 a $(0,14)$ & 1643 & 27,2 a $(0,17)$ & 7,2 & 10 & 0 \\
\hline MONARQUE & 02.08 & 908 & $17,5 \mathrm{c}$ & 33,2 a $(0,16)$ & 1643 & 29,3 a $(0,17)$ & $6,0\left({ }^{1}\right)$ & 20 & 40 \\
\hline INRA 508 & 10.08 & 999 & $18,6 \mathrm{~d}$ & 27,7 a $(0,10)$ & non récolté & 30,6 a $(0,15)$ & - & - & - \\
\hline \multicolumn{10}{|l|}{ Guelph } \\
\hline LG 1 & 01.08 & 807 & $13,8 \mathrm{a}$ & $37,2 \mathrm{a}$ & & & & & \\
\hline SX 111 & 09.08 & 898 & $18,1 \mathrm{c}$ & $52,1 \mathrm{~b}$ & & & & & \\
\hline P 3906 & 16.08 & 966 & $16,3 \mathrm{~b}$ & $44,7 \mathrm{~b}$ & & & & & \\
\hline
\end{tabular}

(1) Les données « récolte " concernant MONARQUE ont été ajustées à partir d'autres essais où figurait également DEA.

TABLEAU $1 \mathrm{~b}$

Observations et caractéristiques agronomiques de l'essai diallèle à Mons. Résultats obtenus pour chacune des combinaisons. Observations and agronomic characteristics of diallel trial at Mons. Results for each combination.

\begin{tabular}{|c|c|c|c|c|c|c|}
\hline & \multicolumn{2}{|c|}{ Floraison } & \multicolumn{4}{|c|}{ Récolte (13 octobre 1983) } \\
\hline & Date & $\Sigma T>6$ & Nb racines & $\begin{array}{l}\text { Rendement } \\
\text { t/ha }\end{array}$ & $\begin{array}{c}\text { Verse racinaire } \\
\%_{0}\end{array}$ & $\begin{array}{c}\text { Verse pourriture } \\
\underset{\%}{0}\end{array}$ \\
\hline$F 257 \times F 250$ & 23.07 & 756 & $41,7 \mathrm{ab} \quad(0,14)$ & 5,9 & 0 & 48 \\
\hline $\mathrm{F} 2 \times \mathrm{F} 250$ & 25.07 & 786 & $35.3 \mathrm{bcd}(0,20)$ & 6,2 & 10 & 30 \\
\hline F2 $\times$ F257 & 25.07 & 786 & 34,9 bcd $(0,20)$ & 4,6 & 55 & 21 \\
\hline F2 $\times$ F252 & 26.07 & 803 & 28,5 e $\quad(0,15)$ & 6,0 & 45 & 18 \\
\hline $\mathrm{F} 257 \times \mathrm{F} 252$ & 27.07 & 817 & 29,4 de $(0,16)$ & 6,0 & 50 & 12 \\
\hline $\mathrm{F} 257 \times \mathrm{F} 272$ & 27.07 & 817 & 38,9 abc $(0,10)$ & 6,9 & 45 & 6 \\
\hline$F 250 \times F 252$ & 27.07 & 817 & $37,7 \mathrm{abc}(0,18)$ & 6,6 & 0 & 27 \\
\hline$F 250 \times F 272$ & 27.07 & 817 & 37,7 abc $(0,09)$ & 5,5 & 0 & 60 \\
\hline $\mathrm{F} 257 \times \mathrm{F} 244$ & 28.07 & 833 & 33,2 cde $(0,22)$ & 6,4 & 10 & 18 \\
\hline $\mathrm{F} 2 \times \mathrm{F} 244$ & 28.07 & 833 & 32,8 cde $(0,13)$ & 6,1 & 0 & 0 \\
\hline $\mathrm{F} 2 \times \mathrm{F} 272$ & 29.07 & 849 & 36,7 abc $(0,14)$ & 6,4 & 20 & 15 \\
\hline $\mathrm{F} 244 \times \mathrm{F} 250$ & 29.07 & 849 & 38,3 abc $(0,15)$ & 5,6 & 0 & 9 \\
\hline $\mathrm{F} 252 \times \mathrm{F} 272$ & 30.07 & 863 & $40,7 \mathrm{ab} \quad(0,16)$ & 5,2 & 0 & 27 \\
\hline$F 244 \times F 252$ & 03.08 & 914 & 37,7 abc $(0,13)$ & 6,2 & 0 & 0 \\
\hline$F 244 \times F 272$ & 03.08 & 914 & $40,1 \mathrm{ab} \quad(0,15)$ & 5,6 & 0 & 3 \\
\hline
\end{tabular}

$a, b, \ldots$ : Comparaison des moyennes (test de Newman et Keuls) ; les lettres communes indiquent que les moyennes ne sont pas significativement différentes au seuil de probabilité de 0,01 pour le nombre de feuilles et de 0,05 pour le nombre de racines.

Means with letters in common are not significantly different at the 0.01 level of probability for leaf number and at 0.05 level for root number.

( ) : Coefficient de variation.

Coefficient of variation.

avec des sommes de températures du même niveau, mais avec un écart plus élevé à Mons qu'à Guelph (213 degrés jour au lieu de 159). Le nombre de feuilles varie avec la précocité de 13,2 à 18,6 à Mons, de 13,8 à 18,1 à Guelph. Le nombre de racines à la floraison paraît très différent entre lieux : de 25,2 à 33,2 (différence non significative) à Mons et de 37,2 à 52,1 à Guelph. Les différences du nombre de racines entre floraison et récolte à Mons peuvent résulter de la poursuite de l'émission racinaire après floraison (observée sur F7, F2 et INRA 508) et de la variabilité des résultats (coefficient de variation de 0,15 à 0,20 ). Les rendements à Mons varient de 5,2 à 7,2 t/ha. L'hybride « DEA » présente une meilleure résistance à la verse.

Dans l'essai diallèle (tabl. $1 \mathrm{~b}$ ) à Mons, l'étalement de la date de floraison n'est que de 9 jours (128 degrés jour), ce qui est en accord avec le choix d'une gamme de précocité plus restreinte. Le nombre total de racines varie de 28,5 à 41,7 . Le rendement des hybrides varie de 4,6 à $6,9 \mathrm{t} / \mathrm{ha}$. Le pourcentage de verse racinaire varie de 0 à 55 p. 100 et le pourcentage de verse par pourriture de la tige de 0 à $60 \mathrm{p}$. 100. Les valeurs moyennes des lignées en hybride (tabl. 2) sont très comparables sauf en ce qui concerne la verse racinaire (de 2 p. 100 pour F244 et F250 à 32 p. 100 pour F257) et la verse par pourriture des tiges (de 6 p. 100 pour F244 à 35 p. 100 pour F250). Le nombre total de racines varie de 33,6 pour $\mathrm{F} 2$ à 38,8 pour $\mathrm{F} 252$.

\section{B. Distributions du nombre de racines par entre- noud}

\section{Essais variétaux}

Les nombres de racines présentes sur les entrenœuds $2,6,2$ à 5,6 à 8 sont donnés au tableau 3a. La 
TABLEAU 2

Nombre moyen des racines par entre-nœud ou groupe d'entre-nouds : valeur en hybride pour chaque lignée.

Mean root number per internode or group of internodes : hybrid value for each inbred lines.

\begin{tabular}{lccccc}
\hline \hline & $\begin{array}{c}\text { Date de } \\
\text { floraison }\end{array}$ & $\begin{array}{c}\text { Nb total } \\
\text { de racines }\end{array}$ & $\begin{array}{c}\text { Rendement } \\
\text { t/ha }\end{array}$ & $\begin{array}{c}\text { Verse } \\
\text { racinaire } \\
\%\end{array}$ & $\begin{array}{c}\text { Verse } \\
\text { pourriture } \\
\%\end{array}$ \\
\hline F2 & 26.07 & 33,6 & 5,8 & 26 & 17 \\
F257 & 26.07 & 35,5 & 5,9 & 32 & 21 \\
F244 & 30.07 & 36,4 & 6,0 & 2 & 6 \\
F250 & 26.07 & 38,2 & 5,9 & 2 & 35 \\
F252 & 28.07 & 34,9 & 6,0 & 19 & 17 \\
F272 & 29.07 & 38,8 & 5,9 & 13 & 22 \\
\hline
\end{tabular}

distribution de ces nombres de racines a été analysée à l'aide d'un test " chi carré " (DAGNÉLIE, 1970) qui permet d'obtenir la probabilité que les effectifs observés soient ou non différents des effectifs théoriques calculés. Ce test indique donc quelle est la probabilité que les effectifs, pour chaque classe de nombre de racines, soient affectés par l'hybride pour lequel l'observation est faite.

Il en ressort que des différences significatives entre hybrides existent pour l'ensemble des entre-nœuds ou groupes d'entre-nœuds étudiés, tant au niveau de la forme des distributions qu'au niveau du nombre de racines qui varie de 3,3 à 4,6 pour $\mathrm{E} 2$, de 13,9 à 19,2 pour E2 à E5, de 5,9 à 10,4 pour E6 et enfin de 13,4 à 32,5 pour E6 à E8. Les chiffres les plus élevés sont toujours obtenus à Guelph sauf pour SX 111 qui a peu de

TABLEAU 3

Nombre moyen de racines allongées par entre-nœud ou groupe d'entre-nœuds.

Mean root number per internode or group of internodes.

$a$ : Essais variétaux de Mons et de Guelph - Varieties studies at Mons and Guelph. $b$ : Essai diallèle de Mons - Diallel trial at Mons.

a - Essai variétaux.

\begin{tabular}{|c|c|c|c|c|c|c|c|c|c|c|}
\hline & \multicolumn{5}{|c|}{ Mons } & \multicolumn{5}{|c|}{ Guelph } \\
\hline & & (') & $(2)$ & $\left({ }^{3}\right)$ & $(4)$ & & $\left({ }^{1}\right)$ & $(2)$ & $\left({ }^{3}\right)$ & (4) \\
\hline \multirow{4}{*}{$\mathrm{E} 2$} & INRA 508 & 135 & 3,3 & $(0,18)$ & $\mathrm{a}$ & SX 111 & 119 & 3,3 & $(0,18)$ & $\mathbf{a}$ \\
\hline & DEA & 134 & 3,6 & $(0,19)$ & $b$ & P 3906 & 100 & 4,2 & $(0,26)$ & $\mathrm{b}$ \\
\hline & MONARQUE & 157 & 3,8 & $(0,18)$ & $\mathrm{c}$ & LG 1 & 98 & 4,6 & $(0,24)$ & $\mathrm{c}$ \\
\hline & $\mathrm{F} 7 \times \mathrm{F} 2$ & 180 & 3,9 & $(0,23)$ & $\mathrm{c}$ & & & & & \\
\hline \multirow{4}{*}{ E2 à E5 } & $\mathrm{F} 7 \times \mathrm{F} 2$ & 139 & 13,9 & $(0,11)$ & a & SX 111 & 60 & 15,9 & $(0,10)$ & $a$ \\
\hline & $\mathrm{DEA}$ & 100 & 14,0 & $(0,12)$ & $\mathrm{a}$ & P 3906 & 56 & 17,3 & $(0,08)$ & $\mathrm{b}$ \\
\hline & INRA 508 & 100 & 14,5 & $(0,16)$ & $a b$ & LG 1 & 58 & 19,2 & $(0,10)$ & $\mathrm{c}$ \\
\hline & MONARQUE & 115 & 14,6 & $(0,10)$ & $\mathrm{b}$ & & & & & \\
\hline \multirow{4}{*}{ E6 } & $\mathrm{F} 7 \times \mathrm{F} 2$ & 100 & 5,9 & $(0,27)$ & $\mathrm{a}$ & LG 1 & 75 & 9,1 & $(0,18)$ & $\mathrm{a}$ \\
\hline & INRA 508 & 60 & 6,3 & $(0,23)$ & $\mathrm{a}$ & P 3906 & 89 & 10,3 & $(0,16)$ & $b$ \\
\hline & MONARQUE & 80 & 6,4 & $(0,22)$ & $\mathrm{a}$ & SX 111 & 20 & 10,4 & $(0,14)$ & $\mathrm{b}$ \\
\hline & DEA & 60 & 7,1 & $(0,22)$ & $\mathrm{b}$ & & & & & \\
\hline \multirow{4}{*}{$\begin{array}{l}\text { E6 à E7 } \\
\text { ou E8 }\end{array}$} & $\mathrm{F} 7 \times \mathrm{F} 2$ & 53 & 13,4 & $(0,38)$ & a & LG 1 & 57 & 21,6 & $(0,12)$ & $\mathrm{a}$ \\
\hline & INRA 508 & 40 & 14,1 & $(0,26)$ & $a b$ & P 3906 & 59 & 23,1 & $(0,11)$ & $\mathrm{b}$ \\
\hline & MONARQUE & 40 & 15,8 & $(0,30)$ & b & SX 111 & 28 & 32,5 & $(0,09)$ & $\mathrm{c}$ \\
\hline & DEA & 40 & 15,9 & $(0,34)$ & b & & & & & \\
\hline
\end{tabular}

b - Essai diallèle de Mons.

\begin{tabular}{|c|c|c|c|c|c|c|c|c|c|c|c|c|}
\hline & \multicolumn{3}{|c|}{$\mathrm{E} 2$} & \multicolumn{3}{|c|}{ E2 à E5 } & \multicolumn{3}{|c|}{ L6 } & \multicolumn{3}{|c|}{ E6 à E8 } \\
\hline & $\left({ }^{2}\right)$ & $\left({ }^{3}\right)$ & $\left({ }^{4}\right)$ & & & & & & & & & \\
\hline $\mathrm{F} 257 \times \mathrm{F} 250$ & 4,0 & $(0,20)$ & bcd & 17,1 & $(0,11)$ & def & 7,3 & $(0,20)$ & $b c$ & 24,7 & $(0,25)$ & $\mathrm{c}$ \\
\hline $\mathrm{F} 2 \times \mathrm{F} 250$ & 3,8 & $(0,21)$ & abcd & 15,5 & $(0,08)$ & $\mathrm{cd}$ & 5,8 & $(0,23)$ & $a b$ & 19,9 & $(0,33)$ & $a b c$ \\
\hline F2 $\times$ F257 & 3,7 & $(0,26)$ & abcd & 15,3 & $(0,28)$ & bcd & 9,4 & $(0,43)$ & d & 19,5 & $(0,30)$ & $a b c$ \\
\hline $\mathrm{F} 2 \times \mathrm{F} 252$ & 3,2 & $(0,23)$ & a & 13,1 & $(0,12)$ & $\mathrm{a}$ & 6,5 & $(0,34)$ & $a b c$ & 15,5 & $(0,30)$ & a \\
\hline $\mathrm{F} 257 \times \mathrm{F} 252$ & 3,4 & $(0,14)$ & $a b$ & 13,5 & $(0,16)$ & $a b$ & 5,1 & $(0,47)$ & a & 15,9 & $(0,26)$ & $a b$ \\
\hline $\mathrm{F} 257 \times \mathrm{F} 272$ & 4,3 & $(0,15)$ & $\mathrm{a}$ & 16,6 & $(0,09)$ & cdef & 6,9 & $(0,16)$ & $a b c$ & 22,3 & $(0,18)$ & bc \\
\hline$F 250 \times F 252$ & 3,9 & $(0,29)$ & bcd & 16,4 & $(0,08)$ & cdef & 5,8 & $(0,15)$ & $a b$ & 22,0 & $(0,31)$ & $a b c$ \\
\hline $\mathrm{F} 250 \times \mathrm{F} 272$ & 4,3 & $(0,19)$ & d & 18,6 & $(0,08)$ & $\mathrm{f}$ & 7,3 & $(0,20)$ & bc & 19,0 & $(0,14)$ & $a b c$ \\
\hline F257 $\times$ F244 & 3,5 & $(0,22)$ & $a b c$ & 16,0 & $(0,16)$ & cde & 7,9 & $(0,22)$ & ed & 17,2 & $(0,41)$ & $a b$ \\
\hline $\mathrm{F} 2 \times \mathrm{F} 244$ & 3,8 & $(0,19)$ & abcd & 14,3 & $(0,12)$ & $a b c$ & 5,7 & $(0,28)$ & $a b$ & 18,5 & $(0,23)$ & $a b c$ \\
\hline F2 $\times$ F 272 & 3,9 & $(0,26)$ & bcd & 16,4 & $(0,08)$ & cdef & 7,1 & $(0,26)$ & $b c$ & 20,3 & $(0,24)$ & $a b c$ \\
\hline $\mathrm{F} 244 \times \mathrm{F} 250$ & 4,0 & $(0,14)$ & bcd & 16,6 & $(0,07)$ & cdef & 5,8 & $(0,13)$ & $a b$ & 21,7 & $(0,25)$ & $a b c$ \\
\hline $\mathrm{F} 252 \times \mathrm{F} 272$ & 3,8 & $(0,21)$ & abcd & 16,7 & $(0,10)$ & def & 6,3 & $(0,28)$ & $a b c$ & 24,0 & $(0,27)$ & $\mathrm{c}$ \\
\hline $\mathrm{F} 244 \times \mathrm{F} 252$ & 4,2 & $(0,29)$ & cd & 15,8 & $(0,12)$ & cde & 5,0 & $(0,21)$ & a & 21,9 & $(0,25)$ & $a b c$ \\
\hline $\mathrm{F} 244 \times \mathrm{F} 272$ & 4,1 & $(0,31)$ & bcd & 17,9 & $(0,14)$ & ef & 6,5 & $(0,24)$ & $a b c$ & $22, \mathrm{I}$ & $(0,30)$ & bc \\
\hline
\end{tabular}

(1) Effectif de la population ; number of plant under study.

(2) Valeur moyenne; mean value.

(3) Coefficient de variation; coefficient of variation.

(4) Comparaison des moyennes (test de Newman et Keuls), les lettres communes indiquent que les moyennes ne sont pas significativement différentes au seuil de probabilité de 0,05 . Means with letters in common are not significantly different at the level 0.05 of probability. 
racines sur l'entre-nœud 2. La variabilité intra-variété, estimée par le coefficient de variation (rapport de l'écart-type sur la moyenne), est assez élevée pour chaque entre-nœud (de l'ordre de 0,20 pour E2 par exemple) et diminue pour les groupes d'entre-nœuds (par l'augmentation de l'effectif) à l'exception de E6 à E8 à Mons. Pour ce lieu, les différences entre génotypes vont en diminuant avec le niveau des entre-nœuds. Le test de NEWMAN et KEULS ne permet plus de distinguer que 2 groupes de génotypes au lieu de 3 lorsque l'on passe de E2 à E6.

Pour le groupe d'entre-nœuds E6 à E8 l'importance des coefficients de variation (de l'ordre de 0,30 ) diminue la puissance du test et ne permet peut-être plus de mettre les différences entre génotypes en évidence. A Guelph, les différences subsistent sur la plupart des entre-nœuds ; ceci peut résulter des plus grands écarts entre les valeurs moyennes observées sur ce site (sauf sur E6) et aussi du fait que la variabilité intra-variété reste plus faible. Il faut remarquer aussi que cette variabilité est du même ordre que celle observée pour un génotype donné cultivé dans différents milieux (JORDAN et al., 1987).

Il apparaît que pour l'espèce maîs le nombre de racines par entre-nœud varie d'un entre-nœud à l'autre alors que pour d'autres graminées (blé, dactyle) il semble constant (PINTHUS, 1969 ; KLEPPER et al., 1984 ; GOUNOT et al., 1980). Ce nombre dépend aussi du génotype et cette liaison est peut-être plus étroite pour les premiers entre-nœuds.

Il est difficile d'établir, à partir de ces résultats, dans quelle mesure cette variabilité génétique résulte d'un «effet précocité »; celui-ci pouvant être différent d'un entre-nœud à l'autre ; ainsi les variétés les plus précoces (F7, F2 à Mons et LG 1 à Guelph) présentent un plus petit nombre de racines que les autres génotypes aux entre-nœuds supérieurs. Il serait intéressant de comparer, sur une gamme de génotypes plus large, la vigueur au départ avec le nombre de racines sur les premiers entre-nœuds et la précocité.

Ces différences de comportement entre variétés semblent montrer des compensations entre les différents entre-nœuds qui n'apparaissaient pas sur les observations effectuées précédemment en différents milieux (JORDAN et al., 1988), et qui peuvent expliquer ici que le nombre total de racines varie peu avec le génotype au moins à Mons (tabl. 1a). Pour Guelph, on peut remarquer que la plus grande différence observée sur SX 111 résulte surtout de l'émission racinaire sur un entre-nœud supplémentaire et qu'il n'y a pas eu d'observations après la floraison alors que l'émission racinaire peut se poursuivre (PICARD et al., 1985).

\section{Essai diallèle}

L'apparentement entre certaines lignées n'empêche pas cette analyse. En effet, ces lignées apparentées présentent des niveaux très différents pour les caractères qui nous intéressent, essentiellement nombre de racines et résistance à la verse. Par ailleurs, la vigueur des hybrides (exprimée par le rendement et la précocité) est très homogène et ne biaise pas les resultats. Seul l'hybride F2 $\times$ F257 (tabl. 1b) a un rendement plus faible, mais c'est le seul hybride corné du diallèle et il est impossible de trouver à ce niveau de précocité des hybrides cornés plus vigoureux.
Les nombres moyens de racines par entre-nœud ou groupe d'entre-nœuds sont donnés dans le tableau $3 \mathrm{~b}$. Sur E2 les moyennes comprises entre 3,2 et 4,3 se distribuent selon 4 groupes qui se recouvrent largement. Les valeurs obtenues pour E2 à E5 varient de 13,1 à 18,6 et peuvent être séparées en 6 groupes, ce qui correspond à la plus forte dispersion observée. On peut séparer 4 groupes de moyennes sur E6 (entre 5,0 et 9,4) et seulement 3 pour l'ensemble des entre-nœuds supérieurs (entre 15,5 et 24,7 ).

Les nombres de racines plus faibles sur E2 à E5 comme sur E6 à E8 sont observés sur F2 $\times$ F252 et F257 $\times$ F252. Pour l'ensemble des génotypes la relation entre nombre de racines sur les premiers entrenœuds (E2 à E5) et les entre-nœuds supérieurs (E6 à E8) est bonne $\left(r=0,67^{* *}\left({ }^{1}\right)\right)$. Par contre la relation est nulle entre $\mathrm{E} 2$ et $\mathrm{E} 6(\mathrm{r}=0,07)$.

Il n'apparaît donc pas ici de compensation importante entre les différents niveaux d'entre-nœuds.

Les nombres de racines des hybrides constitués avec F2 et F252 sont en moyenne plus faibles ; ceux obtenus avec F250 et F272 sont les plus élevés (tabl. 4).

TABLEAU 4

Nombre moyen des racines par entre-noud ou groupe d'entre-nouds : valeur en hybride pour chaque lignée.

Mean root number per internode or group of internodes : hybrid value for each inbred lines.

\begin{tabular}{lccccc}
\hline \hline & E2 & E2 à E5 & E6 & E6 à E8 & Total \\
& & & & & \\
F2 & 3,7 & 14,9 & 7,1 & 18,7 & 33,6 \\
F257 & 3,8 & 15,7 & 7,2 & 19,9 & 35,6 \\
F252 & 3,7 & 15,1 & 5,7 & 19,9 & 35,0 \\
F244 & 3,9 & 16,1 & 6,1 & 20,3 & 36,4 \\
F250 & 4,0 & 16,8 & 6,4 & 21,5 & 38,3 \\
F272 & 4,1 & 17,2 & 6,8 & 21,5 & 38,7 \\
\hline
\end{tabular}

La décomposition de la variance entre Aptitude Générale à la Combinaison (A.G.C.) et Aptitude Spécifique à la Combinaison (A.S.C.) peut être faite selon la méthode de GRIFFING simplifiée (pas d'hybrides réciproques ni de lignées, DEMARLY, 1977). Elle montre que la variance due à l'A.G.C. est significativement plus importante que celle qui est due à l'A.S.C. pour E2 à E5. Par contre pour E6 à E8, l'A.S.C. est aussi importante que l'A.G.C. Cela signifie que le nombre de racines entre E6 et E8 ne peut pas être prévu en fonction de la valeur moyenne en hybride des parents, mais dépend de chaque combinaison.

L'étude de la liaison entre verse et nombre de racines montre une liaison faible mais significative entre le pourcentage de verse racinaire et le nombre total de racines $\left(r=-0,58^{*}\right)$, le nombre de racines entre $\mathrm{E} 2$ et E5 $\left(r=-0,58^{*}\right)$ et le nombre de racines entre E6 et E8 $\left(r=-0,51^{*}\right)$. La taille des hybrides est homogène et $n$ 'interfère pas dans ces liaisons. Les relations avec la verse par pourriture des tiges et la verse totale ne sont pas significatives.

Ces relations nombre de racines-verse racinaire apparaissent logiques et même si elles ne sont pas très

(1) Les seuils de signification sont indiqués comme suit : * pour 0,$05 ;{ }^{* *}$ pour 0,01 . 
étroites, encourageantes pour le sélectionneur d'autant que l'importance de l'A.G.C. pour le nombre de racines sur les premiers entre-nœuds permet d'envisager une sélection au stade jeune indépendante du testeur. Cependant, on peut se demander aussi s'il ne faut pas rechercher d'autres paramètres que ce nombre de racines. Les racines primaires et leurs ramifications définissent un certain volume autour de la partie souterraine de la tige ( (bol racinaire ») dont l'importance et la densité des racines sont certainement plus en relation avec la verse racinaire.

\section{Cinétique de mise en place du système racinaire}

Seuls les essais variétaux sont étudiés.

\section{En fonction des sommes de températures de l'air}

Les sommes des températures permettent de décrire le développement du maïs (BLOC \& GOUET, 1978; DERIEUX \& BONHOMME, 1982). Elles ont été utilisées aussi par BLOC et al. (1979) pour décrire l'apparition des feuilles chez différents hybrides en différents lieux.

Dans notre étude, l'ajustement linéaire nombre de racines-somme des températures au-dessus du seuil $6{ }^{\circ} \mathrm{C}$ donne des résultats satisfaisants (fig. 1).

Pour chaque lieu, une variété apparaît significativement différente des autres (DEA à Mons et SX 111 à Guelph) mais ces différences de pente résultent seule-

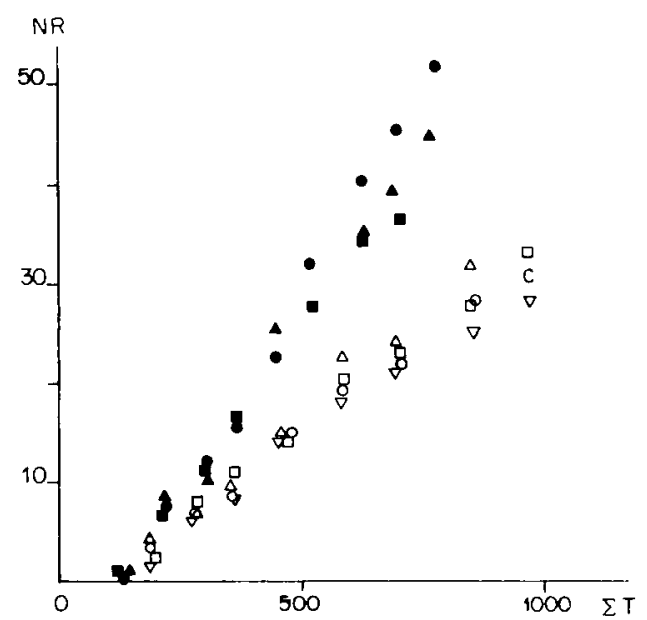

Figure 1

Emission racinaire: nombre de racines primaires allongées (NR) en fonction des sommes de température de l'air seuil $6{ }^{\circ} \mathrm{C}(\Sigma T)$ reçues depuis le semis.

Root appearance : number of primary roots $(N R)$ in relation to temperature sum (degree-days) from sowing.

\section{Guelph}

a $L G 1: N R=0,067 \Sigma T-7,9 \quad r=0,99 * * *(1)$

- $S X 111: N R=0,079 \Sigma T-10,2 \quad r=0,99 * * *(2)$

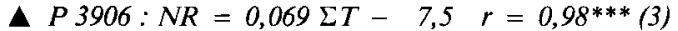

Test de parallélisme : pente (2) significativement différente au seuil 0,05 .

Slope (2) significantly different at level 0.05 .

Mons

$\square$ MONARQUE: NR $=0,038 \Sigma T-3,5 \quad r=0,99 * * *(I)$

OINRA 508 $: N R=0,035 \Sigma T-3,3 \quad r=0,99 * * *(2)$

$\therefore D E A \quad: N R=0,044 \Sigma T-5,7 \quad r=0,98 * * *$ (3)

$\nabla F 7 \times F 2 \quad: N R=0,035 \Sigma T-3,5 \quad r=0,98^{* * *}(4)$

Test de parallélisme : pente (3) significativement différente au seuil 0,05 .

Slope (3) significantly different at level 0.05 ment de celles observées à deux dates et il n'est pas certain que sur un plus grand nombre d'observations ou de traitements elles subsistent. Les écarts entre les deux sites sont très importants et correspondent surtout (fig. 2) à un nombre de racines plus grand sur chaque entre-nœud des variétés cultivées à Guelph.

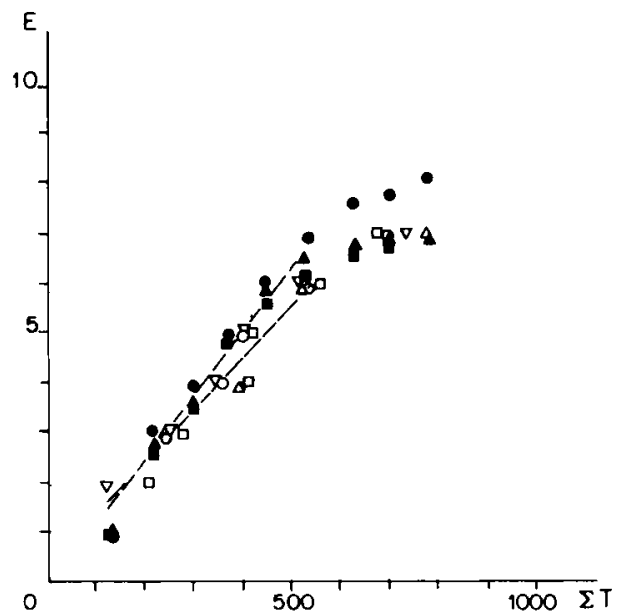

Figure 2

Emission racinaire: nombre moyen d'entre-nœuds (E) possédant $50 \%$ des racines allongées en fonction des sommes de températures de l'air seuil $6{ }^{\circ} \mathrm{C}(\Sigma T)$ reçues depuis le semis.

Root appearance : number of internodes $(E)$ with $50 \%$ of roots in relation to temperature sum (degree-days) from sowing.

Guelph

- $L G 1 \quad E=0,0124 \Sigma T-0,01$ (I) $\quad r=0,98^{* * *}$

- SX 111 (Test de parallélisme : pas de différence

A P3906 significative entre génotype)

Mons

$\nabla F 7 \times F 2 \quad E=0,0105 \Sigma T+0,33(2) \quad r=0,97^{* * *}$

$\triangle D E A \quad$ (Test de parallélisme : pas de différence

INRA 508 significative entre génotype)

$\square$ MONARQUE

Les pentes (1) et (2) sont significativement différentes au seuil 0,01 .

En effet, l'émission des racines par entre-nœud diffère peu entre Guelph et Mons (fig. 2) même si cette différence (sur l'ajustement des parties linéaires) est significative. Celle-ci résulte de la mise en place de racines sur un niveau supplémentaire (E8) pour l'une des variétés (SX 111) et de la valeur des sommes de températures relativement basse pour obtenir 50 p. 100 des racines du premier entre-nœud.

La température de l'air n'est qu'un indicateur de celle au niveau des racines; la relation entre ces deux paramètres dépend des caractéristiques du sol et des masses d'air et peut donc être différente entre les deux sites. Cela pourrait expliquer au moins une part des écarts observés entre les deux lieux.

\section{En fonction du nombre de feuilles}

A Mons, dans la phase d'émission active des feuilles (fig. 3), on obtient une relation linéaire entre le nombre de racines allongées (NR) et le nombre de feuilles visibles (NF).

Peu avant la floraison et conformément aux résultats antérieurs (PICARD et al., 1985) il y a pratiquement arrêt de l'émission foliaire alors que l'émission racinaire se poursuit sur les entre-nœuds supérieurs à 6 . Le nombre des points correspondant à cette phase finale 


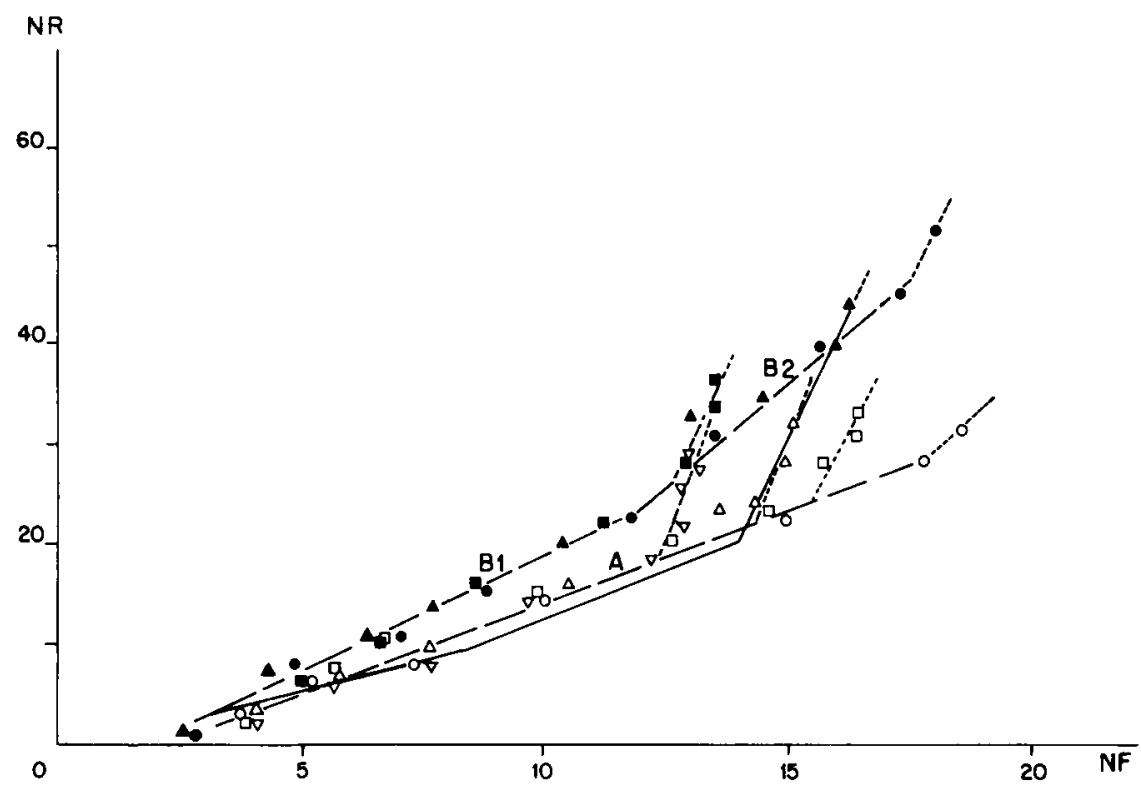

Figure 3

Emission racinaire : nombre de racines primaires allongées (NR) en fonction du nombre de feuilles visibles (NF).

Root appearance of primary roots (NR) in relation with number of visible leaves (NF).

$\begin{array}{ll}\text { Mons } & \text { Guelph } \\ \nabla F 7 \times F 2 & \text { - LG I } \\ \triangle D E A & \bullet S X 111 \\ \bigcirc I N R A 508 & \text { \ } P 3906\end{array}$

$\square$ MONARQUE

n'est jamais suffisant pour calculer un ajustement; la droite n'est donnée qu'à titre indicatif (petits pointillés sur la fig. 3). Si on ne prend pas en considération cette dernière phase, on constate que le modèle linéaire est unique pour toutes les variétés et présente une pente voisine de la pente moyenne calculée sur DEA à Colmar par PICARD et al. (1985).

A Guelph, le nombre de racines allongées est toujours plus important. Jusqu'au stade 12-13 feuilles, les pentes des ajustements linéaires entre NR et NF ne diffèrent pas significativement entre les 3 variétés. Après ce stade la pente est plus forte et de même valeur pour SX 111 et P 3906 alors que la variété LG 1 entre en phase finale d'émission racinaire.

Comme il n'y a pas de variété commune aux deux expérimentations, il n'est pas possible d'attribuer les différences de pentes observées entre les génotypes de Guelph et ceux de Mons à la variabilité génétique ou à un effet du milieu. Cependant, la variété LG 1 est un hybride français connu et génétiquement comparable à la variété DEA ou aux hybrides F2 $\times$ F244 et F2 $\times$ F252 étudiés à Mons. Aussi les différences de comportement entre les deux sites, au moins pour LG 1 , correspondraient plus à un effet du milieu.

Les émissions racinaire et foliaire dépendent de la température du sol et de l'air selon des lois différentes (BONHOMME, 1983). Les écarts de température entre le sol et l'air peuvent varier avec le site (nature du sol, régime des masses d'air...) et pourraient peut-être expliquer en partie cet effet du milieu.

Cependant les pentes obtenues pour SX 111 et P 3906 après le stade 12-13 feuilles sont très fortes et cela traduirait peut-être des aptitudes propres à ces variétés sélectionnées au Canada.

Enfin pour tous les génotypes et les deux sites, on observe une pente nettement plus forte peu avant la floraison qui paraît assez peu variable et de même valeur que celle calculée sur DEA à Colmar (PICARD et al., 1985).

\section{CONCLUSION}

L'approche expérimentale utilisée ici et dans nos travaux précédents (PICARD et al., 1985) et suivants (JORDAN et al., 1988) ne permet pas d'analyser les interactions génotype $\times$ milieu mais seulement d'explorer une certaine expression de la variabilité de l'émission racinaire en conditions naturelles.

Cette étape nous paraissait indispensable avant une analyse plus fine de la mise en place du système racinaire.

La structuration d'un pied de maïs comme un empilement de phytomères : unité morphologique composée d'un entre-nœud surmonté d'un nœud, d'une feuille et de son bourgeon axillaire et d'une série d'ébauches racinaires à la base de l'entre-nœud (KISSELBACH, 1949 ; GIRARDIN et al., 1986), laisse prévoir une coordination entre les émissions racinaire et foliaire. Celle-ci se traduit par une relation étroite entre le nombre total de racines allongées et le nombre de feuilles visibles qui peut être exprimée par la succession de 2 ou 3 ajustements linéaires. Cette représentation s'applique à tous nos traitements (génotypes- 
milieux) et pourrait être représentative non seulement du maïs mais d'autres graminées (riz, blé...).

Ces relations sont intéressantes puisqu'elles permettent de déterminer une caractéristique du système racinaire à partir du nombre de feuilles : paramètre facile à mesurer ou à modéliser (relation avec la somme des températures de l'air, cf. BONHOMME, 1983 par exemple). Leur stabilité reste néanmoins à vérifier au vu des résultats obtenus à Guelph. De plus, bien que les vitesses d'apparition soient très liées, le nombre final de racines ne peut être prévu à partir de celui des feuilles en raison de la grande variabilité du nombre de racines émises sur les derniers entre-nœuds.

A l'issue de ces travaux il semble que l'action du milieu sur le nombre de racines allongées par entrenœud soit de plus en plus forte avec le numéro de l'entre-nœud et plus particulièrement après E6. Ces résultats posent le problème de la signification du nombre de racines allongées notamment pour les entrenœuds supérieurs pour lesquels des racines restent à l'état d'ébauches, et du numéro du dernier entre-nœud présentant des racines allongées. Ces relations ne semblent pas pouvoir être reliées pour un génotype donné avec le nombre de feuilles. Elles pourraient traduire des phénomènes de compétition entre le système racinaire et les autres organes pour l'utilisation des assimilats au tout début de la phase de maturation. Ceci expliquerait aussi un effet de plus en plus marqué du milieu, même si, au niveau de cette régulation il peut y avoir interaction avec le génotype. Il serait donc particulièrement intéressant d'étudier la mise en place du système racinaire en fonction de l'alimentation carbonée de la plante.

Recu le 13 août 1986. Accepté le 12 juin 1987.

\section{RÉFÉRENCES BIBLIOGRAPHIQUES}

Barrière Y., 1979. Sélection du maïs pour la résistance à la pourriture des tiges. Etudes de génotypes précoces. Ann. Amélior. Plant., 29 (3), 289-304.

Bloc D., Gouet J. P., 1978. Influence des sommes de température sur la floraison et la maturité du maïs. Ann. Amélior. Plant., 28 (1), 89-112.

Bloc D., Bonhomme R., Gay J. P., Girardin P., Gouet J. P., 1979. Etude de l'influence de la température sur le développement du maïs. Résultats 1978-1979. A.G.P.M., 34 p.

Bonhomme R., 1983. Mise en place des appareils foliaire et racinaire. In Physiologie du maïs. Coll. I.N.R.A., C.N.R.S., A.G.P.M., ed. A. Gallais, Royan, 15-17 mars 1983, 63-83.

Dagnélie P., 1970. Théorie et méthodes statistiques. Vol. 2, Presses. Agron. Gembloux, Edit. J. Duculot, 463 p.

Demarly Y., 1977. Génétique et amélioration des plantes. Ed. Masson, $287 \mathrm{p}$.

Derieux M., 1978. Etude de quelques facteurs d'adaptation du mais aux conditions climatiques du nord de la France. Ann. Amélior. Plant., 28 (5), 529-566.

Derieux M., Bonhomme R., 1982. Heat unit requirements for maize hybrids in Europe. Results of the European F.A.O. subnetwork. I. Sowing-silking period. Maydica, XXVII, 59-77.

Gounot M., Atry M., N'Kandza J., Yu O., 1980. Photosynthèse nette et rythme d'apparition des feuilles chez le dactyle (Dacty/is glomerata L.). C. R. Acad. Sci., Paris, 290 D, 1257-1260.
Girardin Ph., Jordan M. O., Picard D., Trendel R., 1986. Harmonisation des notations concernant la description morphologique d'un pied de maïs. Agronomie, 6 (9), 873-875.

Jordan M. O., Girardin Ph., Varlet-Grancher C., Picard D., Trendel R., 1988. Rythme d'apparition des racines primaires du maïs (Zea mays L.). III. Variations observées au champ. Agronomie, 8 (1)

Kiesselbach T. A., 1949. The structure and reproduction of corn. Univ. of Nebraska Press, reed., 1980, 96 p.

Klepper B., Belford R. K., Rickman R. W., 1984. Root and development in winter wheat. Agron. J., 76, 117-122.

Onderdonk J., Ketcheson J. W., 1972. A standardization of terminology for the morphological description of corn seedlings. Can $J$. Plant Sci., 52, 1003-1006.

Picard D., 1984. Etudes racinaires et résistance à la sécheresse. Comm. Colloque «Résistance à la sécheresse en milieu tropical . Quelles recherches à moyen terme ? ». Dakar, 24-27/09/1984, 131143.

Picard D., Jordan M. O., Trendel R., 1985. Rythme d'apparition des racines primaires du maïs (Zea mays L.). I. Etude détaillée pour une variété en un lieu donné. Agronomie, 5 (8), 667-676.

Pinthus M. J., 1969. Tillering and coronal root formation in some common and durum wheat varieties. Crop Sci., 9, 267-272. 